\title{
Tree Physiology Optimization tuning rule for Proportional-Integral control
}

\author{
A.Hanif Halim*, and $I$. Ismail \\ Electrical and Electronic Engineering Department, Universiti Teknologi PETRONAS, \\ 31750 Tronoh, Perak, Malaysia
}

\begin{abstract}
This paper presents a tuning correlation for ProportionalIntegral (PI) controller parameters using Tree Physiology Optimization algorithm (TPO). TPO is a metaheuristic algorithm that has parallel search strategy inspired from plant growth system. The parallel search is referred to the plant branches and leaves that able to diversify the search space and therefore finding promising results in much faster iterations. The exploration is amplified from roots growth counterparts. In the proposed method, a tuning correlation of PI-controller using TPO is established and the performances of correlated parameters are compared with Ciancone correlation. The idea of correlation tuning is to reduce the optimization time consumption and engineering efforts in controller tuning. The results shown that the tuning correlation of TPO is able to find good PI parameters with lesser error.
\end{abstract}

\section{Introduction}

PI and PID controller is often used in industries and is acknowledged as an effective control system in numerous controlling applications. The unique properties of each controller part: proportional, integral and derivative made the controller as a standard controlling tool that should be able to reduce the errors with good set point tracking and disturbance rejection within a desirable tolerance. The tuning of control parameters is depending heavily on plant properties such as order of the system, plant gain, time constants and dead time [1]. In practice, other factor such as process interruption is also included in finding the optimum PID parameter. To date, there are numerous tuning rule and optimization approach of such problems. This includes the traditional methods: Ziegler-Nichols (ZN) method that based on integral of absolute error index (ISE) [2] Tyreus-Luyben (TL) [3], and Ciancone correlation [4]. Other proposed optimization approaches are using metaheuristic algorithm such as Particle Swarm Optimization (PSO), Genetic Algorithm (GA), and many more [5-9]. However, optimization search with certain defined of iteration number is time consuming and improper optimization parameter may result in poor controller performance [10]. In practice, it is sufficient to obtain the controller parameter that able to perform within defined response characteristic without performing an optimization task. In this paper, a tuning correlation of PI-controller parameter based on

*Corresponding author: abdul_g01772@utp.edu.my 
TPO algorithm is proposed. The correlation is defined by each controller parameters: proportional gain (Kp) and Integral time (Tn).

\section{Proposed tuning for PI control}

The proposed tuning rule for PI-control is obtained from TPO search algorithm. TPO is a metaheuristic algorithm developed by A. Hanif Halim and I. Ismail [12]. The algorithm is inspired from a plant growth that consists of two sections: shoots and roots. The shoots section expands their branches and leaves towards light and convert light into Carbon by photosynthesis process. The Carbon gain is then supplied downwards and utilized by the root system for root-growth in search for nutrients. The nutrient gain is then supplied upwards and used by the shoot system for shoots-growth in extension towards light. This relationship of both plant parts towards better growth had inspired the idea of TPO algorithm. The search of TPO has its uniqueness due to the branches system with many shoots that able to search towards optimum in parallel. This is synonym to the normal tree physiological concept. This unique attribute show a better probability of solution due to the ability of more search effort that driven by the best solution found within branch and between branches. The search become broader as the random motion included in each shoot propagation. Several studies on TPO performance and comparison with other algorithm have shown the ability of the algorithm to find the global optimum efficiently. In online PID controller optimization, TPO outperformed other algorithms with faster response and lesser overshoot [9]. TPO is also performed significantly better with lesser computation time than other meta-heuristic algorithm for nonlinear optimization [11, 12]. The idea of tree physiological system is devised into four equations: shoots propagation, carbon gain, root elongation and nutrient uptake. The shoot propagation towards light is shown as in (1):

$$
S_{i}^{k_{j}}=S_{i}^{k_{j}}+\left(S_{g b e s t}-S_{i}^{k_{j}}\right)+\beta N_{i}^{k_{j}}
$$

With $S_{i}^{k_{j}}$ as the current shoot at $\mathrm{i}^{\text {th }}$ iteration, of $\mathrm{k}^{\text {th }}$ shoot and $\mathrm{j}^{\text {th }}$ branch, $S_{\text {gbest }}$ is the global best shoot from all branches, $N_{i}^{k_{j}}$ is the current nutrient supply from root, and $\beta$ is the dynamic factor of shoots extension. Each shoot will undergo the photosynthesis process that converts light into carbon. The carbon gain is equivalent to deviation of individual branch best with individual shoot as in (2):

$$
C_{i}^{k_{j}}=\theta^{i}\left(S_{\text {popbest }}-S_{i}^{k_{j}}\right)
$$

$C_{i}^{k_{j}}$ is current carbon gain, $S_{\text {popbest }}$ is best shoot from current branch. The parameter $\theta$ is a power law formula that reduces the randomness as iteration increases in a monotonically decreasing function. $C_{i}$ amplifies the root elongation in search for more nutrients (3):

$$
r_{i}^{k_{j}}=r_{i}^{k_{j}}+\alpha C_{i}^{k_{j}} \epsilon
$$

$r_{i}^{k_{j}}$ is current root, $\alpha$ is an absorption constant, $\varepsilon$ is the random numbers drawn from uniform distribution. The nutrient uptake can be assumed as a proportional factor to the root elongation. It is expressed in the same formulation as carbon gain

$$
N_{i}^{k_{j}}=\theta\left(r_{i}^{k_{j}}-r_{i 0}^{k_{j}}\right)
$$


These formulations run iteratively until a significant optimum is found by the shoots counterparts. The computation of search process depends on the number of shoots, number of branches and number of iteration. The correlation of optimized PI controller parameter with TPO algorithm is carried out with first-order-with-dead-time transfer function as in (5), since many processes can be represented by this function [10].

$$
G_{s}=\frac{K_{C} e^{-\theta s}}{1+\tau s}
$$

The correlation is established with parameter from fraction-dead-time that is defined with $\theta /(\theta+\tau)[15]$.

\section{Optimization formulation}

The optimum controller parameter determined from TPO is carried out by varying $\theta, \tau:[2,16]$ and $K_{C}:[0.2,3.2]$. The performance criterion to evaluate the performance of the algorithm is based on integral of absolute error (IAE) with conditional programming of response constraint as shown in (1) and Fig. 1.

$$
I A E=\int|e| d t
$$

Based on Fig. 1, $f_{\text {up }}$ and $f_{\text {low }}$ are the upper and lower border of shaded region. $f_{\text {up }}$ consists of two borders $\left(f_{u p}\right.$ and $\left.f_{u p 2}\right), y_{i}$ is the plant response at each iteration.

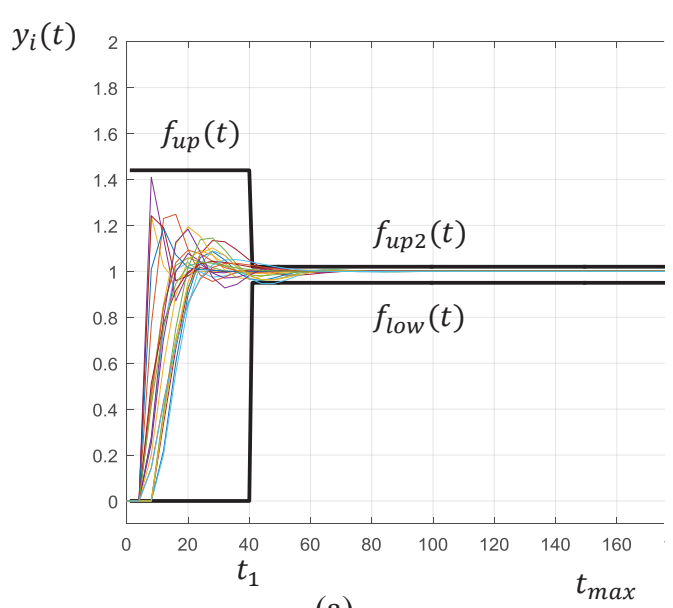

(a)

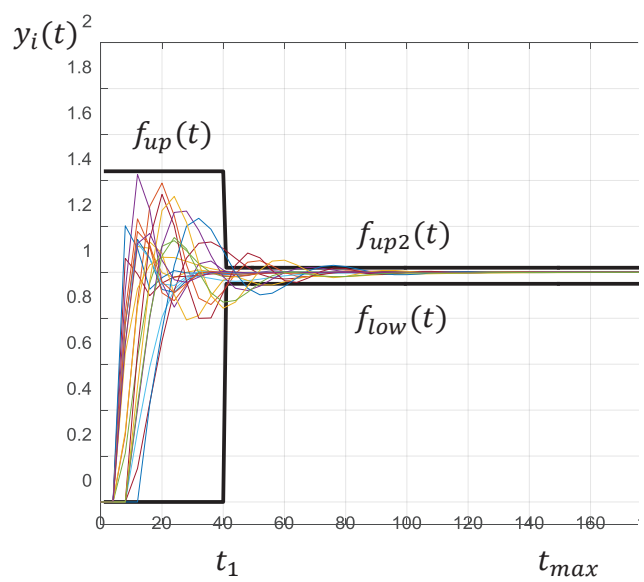

(b)

Fig. 1: Desired region for plant response with (a) lower Kc (b) higher Kc

As observed in the figure, the settings of $f_{u p}(t), f_{\text {low }}(t)$, and $t_{1}$ can be specified by user. In this paper, the response setting is set with $f_{\text {up }}=1.44 ; f_{\text {up } 2}=1.08 ; f_{\text {low }}=0.85 ; t_{1}=$ $20 \% ; t_{\max }=100 \%$. The algorithm run iteratively until it covers all the response value defined for $\theta, \tau, K c$. The parameter setting for TPO in this study is set according to Table 1. 
Table 1: Parameter setting for TPO

\begin{tabular}{|l|l|}
\hline Number of leaves (population) & 10 \\
\hline Number of branch (Sub-population) & 5 \\
\hline Number of iteration & 10 \\
\hline Dynamic factor, $\beta$ & 50 \\
\hline Randomization factor, $\theta$ & 0.9 \\
\hline Absorption factor, $\alpha$ & 0.3 \\
\hline
\end{tabular}

The defined number of branch and leaves show that TPO algorithm is able to search in parallel with possibility solution of $5 \times 10$ in each iteration. A regression correlation between proportional gain and integral time with fractional dead-time is analyzed and depicted in Fig. 2 and Fig. 3 respectively.

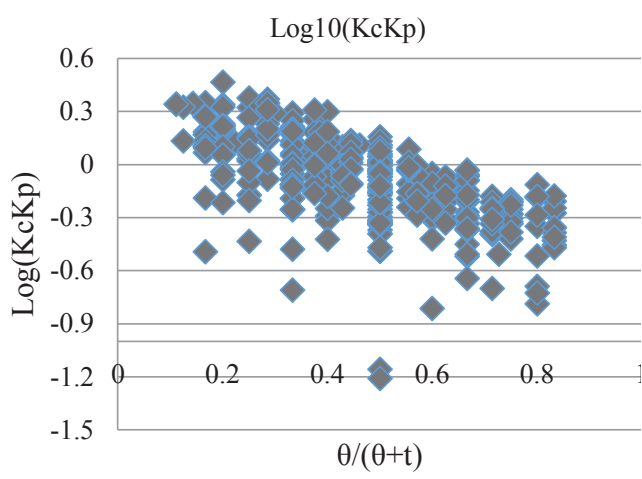

(a)

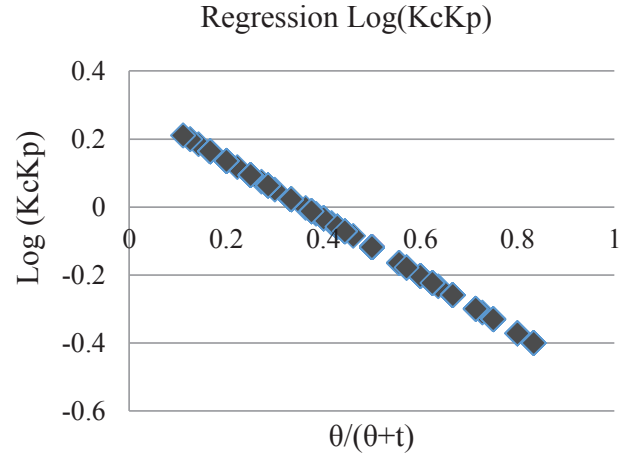

(b)

Fig. 2: Evaluated TPO results for $\mathrm{Kp}$ (a) and regression of $\mathrm{Kp}(\mathrm{b})$

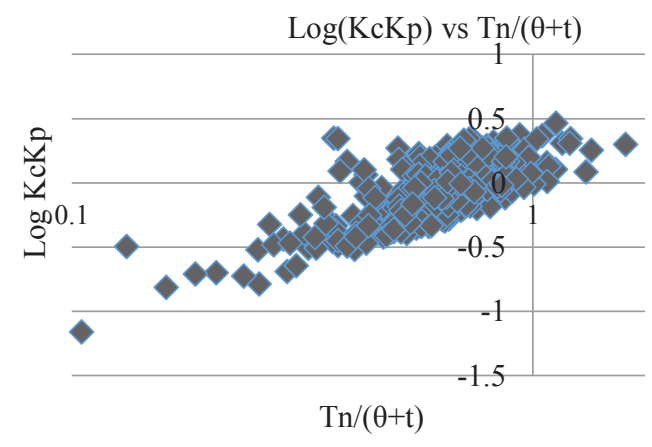

(a)
Regression $\log (\mathrm{KcKp})$ vs $\mathrm{Tn} /(\theta+\mathrm{t})$

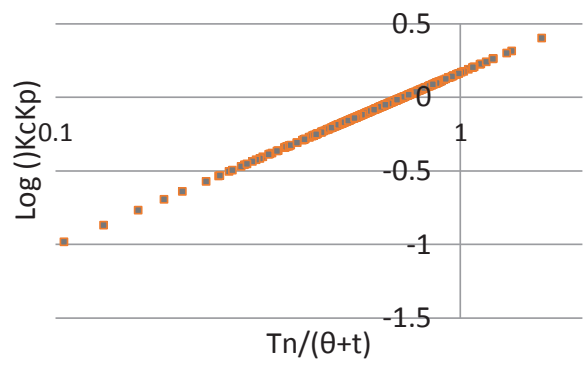

(b)

Fig. 3: Evaluated TPO results for Tn (a) and regression of Tn (b)

\section{Performance comparison}

The correlation performance is compared with Ciancone correlation as defined in [10]. Fig. 4 shows the comparison of step response from TPO and Ciancone correlation. The summary results of each method by different plant parameters are tabulated in Table 2 . 
Comparison for $\mathrm{t}=8, \theta=4, \mathrm{Kc}=2.8$

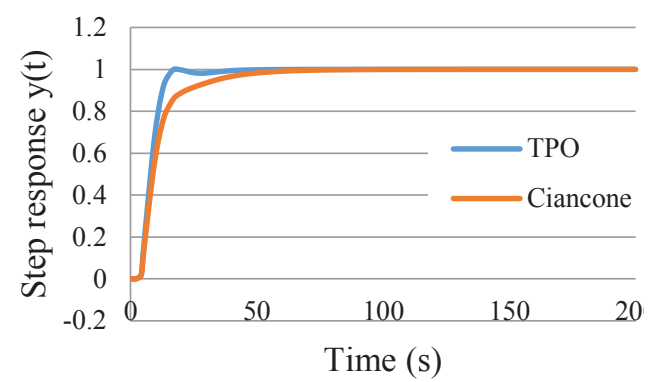

(a)

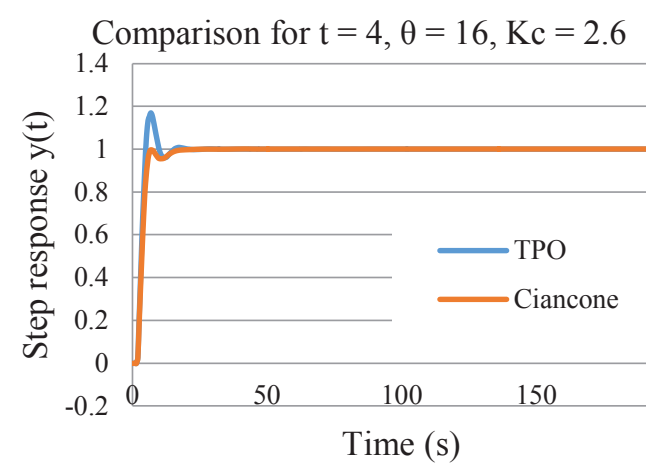

(c)

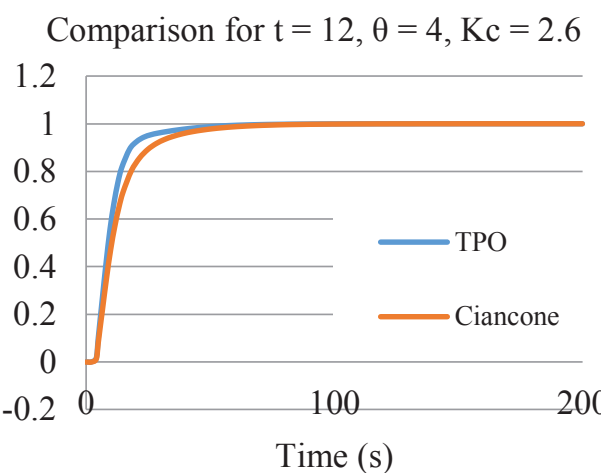

(b)

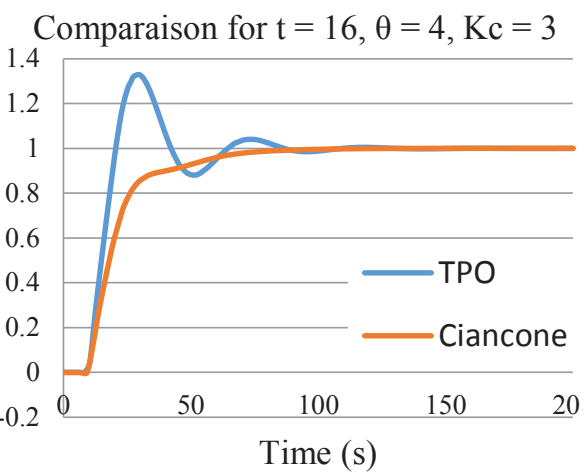

(d)

Fig. 4: Comparison of step response for TPO and Ciancone correlation by different time constant and plant gain

Table 2: Optimum PI of each method

\begin{tabular}{|c|c|c|c|}
\hline Method & $\mathrm{Kp}$ & $\mathrm{Ki}$ & IAE \\
\hline \multicolumn{4}{|c|}{$\mathrm{t}=8, \theta=4, \mathrm{Kc}=2.8$} \\
\hline Ciancone & 0.3214 & 0.0298 & 11.9846 \\
\hline TPO & 3.577 & 0.158 & 8.6475 \\
\hline \multicolumn{4}{|c|}{$\mathrm{t}=12, \theta=4, \mathrm{Kc}=2.6$} \\
\hline Ciancone & 0.388 & 0.0276 & 13.935 \\
\hline TPO & 0.477 & 0.034 & 11.312 \\
\hline \multicolumn{4}{|c|}{$\mathrm{t}=4, \theta=16, \mathrm{Kc}=2.6$} \\
\hline Ciancone & 0.147 & 0.0144 & 26.783 \\
\hline TPO & 0.163 & 0.0239 & 37.431 \\
\hline \multicolumn{4}{|c|}{$\mathrm{t}=16, \theta=4, \mathrm{Kc}=3.0$} \\
\hline Ciancone & 0.286 & 0.0317 & 22.543 \\
\hline TPO & 0.393 & 0.0604 & 22.065 \\
\hline
\end{tabular}

Findings from Fig. 4 and Table 2 shows that TPO has slightly better results in several cases. Ciancone correlation resulted a step performance without overshoot compared to TPO. The performance of TPO can be significantly improved by increasing the number of search space, which is the number of branches and leaves. This can lead the search agents to 
discover more promising solution as the search space become broader. Another possibility of improvement is by tightening the constraints of step response as in Fig. 1.

\section{Conclusion}

In this paper, the correlation of optimum PI controller parameters and first-order-with-deadtime transfer function is established. The correlation is carried out by running TPO algorithm for wide range of dead time, time constant and plant gain. Based on the regression model of each controller parameter with fraction dead-time, a correlation for optimum step response with defined constraints can be evaluated. The performance of evaluated TPO is compared with Ciancone correlation by selecting several step properties. Based on the comparison, Ciancone correlation did shows good results without overshoot, but with slightly higher absolute error (IAE) for three cases. The performance of TPO can be further improved by increasing the number branches and leaves for broader exploration search, and also by tightening the constraints for step response properties. The findings from this paper suggest a necessity of more comparison study by increasing the search capability of TPO and to cover wider range of each plant parameters that include dead-time, time constant and plant gain. The findings also implies a necessity of finding a tuning correlation of set point tracking for PID controller and for both type (PI and PID controller) with disturbance rejection.

The author would like to thank Universiti Teknologi PETRONAS for the support of the paper.

\section{References}

1. K. Ogata, "Modern Control Engineering", $5^{\text {th }}$ Edition Prentice Hall (2010).

2. J. G. Ziegler, N. B. Nichols, "PID Controller: Theory, Design, and Tuning", ISA Research Triangle Park (1995).

3. L. Luyben., "Simple method for tuning SISO controllers in multivariables systems", Industrial \& Engineering Chemistry Process Design and Development, 25(3), pp 654660 (1986)

4. Marlin T. E., "Process Control", $2^{\text {nd }}$ Edition, McGraw-Hill (2000)

5. P. G. Medewar, R. K. Munje, "PSO based PID controller tuning for PMDC motor", Int. Conf. on Energy Systems and Application (2015)

6. P. Ouyang, Vangjel Pano, "Comparative Study of DE, PSO and GA for tuning Position Domain PID Controller", ROBIO (2014)

7. Kelvinder S., Pandian V., Irraivan E., Ramani K., "PID Tuning of Servo Motor using Bat Algorithm, $19^{\text {th }}$ Int. Conf. on Knowledge Based and Intelligent Information and Engineering Systems (2015)

8. Omar B., "Continuous Firefly Algorithm for Optimal Tuning of PID Controller in AVR System", Journal of Electrical Engineering, 65, No. 1, pp 44 - 49 (2014)

9. A. Hanif Halim, I. Ismail, “ Online PID controller tuning using tree physiology optimization", ICIAS, pp 1- 5 (2016)

10. T. Marlin, "Process Control, Designing Processes and Control Systems for Dynamic Performance", $2^{\text {nd }}$ Edition, McGraw-Hill (2015)

11. A. Hanif Halim, I. Ismail, "Comparative Study of Meta-heuristics Optimization Algorithm using Benchmark Function", IJECE, 7, No 2 (2017)

12. A. Hanif Halim, I. Ismail, "Nonlinear plant modeling using neuro-fuzzy system with Tree Physiology Optimization”, (SCOReD) (2013) 